\title{
ON THE STRONG SOLUTION OF A CLASS OF PARTIAL DIFFERENTIAL EQUATIONS THAT ARISE IN THE PRICING OF MORTGAGE BACKED SECURITIES*
}

\author{
RANA D. PARSHAD ${ }^{\dagger}$, DERVIS BAYAZIT ${ }^{\ddagger}$, NATHANIEL S. BARLOW $^{\S}$, \\ AND V. RAMCHANDRA PRASAD
}

\begin{abstract}
We consider a reduced form pricing model for mortgage backed securities, formulated as a non-linear partial differential equation. We prove that the model possesses a weak solution. We then show that under additional regularity assumptions on the initial data, we also have a mild solution. This mild solution is shown to be a strong solution via further regularity arguments. We also numerically solve the reduced model via a Fourier spectral method. Lastly, we compare our numerical solution to real market data. We observe interestingly that the reduced model captures a number of recent market trends in this data, that have escaped previous models.
\end{abstract}

Key words. Mortgage backed security, reduced modeling, mild solution, strong solution, Fourier spectral method.

AMS subject classifications. 35A01, 35D35, 76M22, 91G20, 91G80.

\section{Introduction}

Real world modelling often involves the description of complex and non linear processes, which can be difficult to deal with in their most general forms. One school of thought considers various limiting cases of a parameter or variable of interest in a model, that might lead to a simplification. The equations obtained via these limits, albeit unrealistic, might be easier to analyse or perform numerical computations on. This is particularly favourable in specific scenarios, where there is evidence that actually taking these limits is feasible. Recently this approach has been carried out successfully in analysing partial differential equations describing fluid convection [17], and in analysing partial differential equations describing fluid convection in a porous media [11]. In [11] we proved convergence of global attractors and stationary statistical properties of the Darcy-Boussinesq system to those of the infinite Darcy-Prandtl number model. This model is a reduced variant of the Darcy-Boussinesq system when the permeability (which is a measure of a porous medium to transmit a fluid) approaches 0 . In tightly packed media such as granite, limestone or dolomite, taking this limit is feasible [20]. This makes a strong case for the use of the infinite DarcyPrandtl number model, in applications like hydraulic fracturing or thermal induced oil recovery, in particular if the drilling scenarios are in very tightly packed media $[20]$.

In [12] we started a program where we adopted some of the methodology of the above mentioned works to study mortgage backed securities. Recall that a mortgage backed security is formed by pooling together a group of mortgages and then selling this pool as a security to investors. These constitute over a trillion dollar issuance in

*Received: November 4, 2010; accepted (in revised version): March 19, 2011. Communicated by David Cai.

${ }^{\dagger}$ King Abdullah University of Science and Technology, Applied Mathematics and Computational Science, Thuwal 23955-6900, Kingdom of Saudi Arabia (rana.parshad@kaust.edu.sa).

${ }^{\ddagger}$ Federal Home Loan Bank of Atlanta, 1475 Peachtree Street, N.E. Atlanta, GA 30309, USA.

$\S$ Center for Computational Research, State Universty of New York at Buffalo, Buffalo, NY 14203, USA.

\Department of Mathematics, Madanapalle Institute of Technology and Science, Madanapalle 517325, Andhra Pradesh, India. 
the United States debt markets alone [9], and were the chief cause of the financial crisis that struck the United States markets in 2008 [10]. Given these sordid events, it is beneficial to further understand the price dynamics of MBS. This, however, is a very complicated endeavour due to the difficulties in quantifying prepayment behaviour and measuring the associated risk of these instruments.

In [12] we considered the following non-linear partial differential equation, describing the price evolution $u(x, t)$, of a mortgage backed security and derived in $[13,14,21]$ :

$$
\begin{gathered}
\frac{\partial u}{\partial t}=\frac{1}{2} \Delta u+\mu(\mathbf{x}, t) \nabla u-\rho \frac{\left.\mid \sigma^{T}(\mathbf{x}, t)\right)\left.\nabla u\right|^{2}}{u+h(\mathbf{x}, t)+\xi(t)}-(r(t)-\tau) h(t)-r(t) u \\
(\mathbf{x}, t) \in \mathbb{R}^{N} \times(0, T)
\end{gathered}
$$

Here

$$
r(t)=\delta(T-t), \quad \xi(t)=A_{0} e^{\int_{0}^{T-t} \delta(s) d s}, \quad h(\mathbf{x}, t)=M B(t) e^{-S(\mathbf{x}, t)},
$$

where $\delta$ is a deterministic discount rate and $\mathbf{x}$ are the various economic factors that the price of a MBS could depend on, such as interest rates. $S(\mathbf{x}, t)$ is the so called prepayment function $^{1}$, for which there is no closed form and is therefore constructed empirically, [9].

Note that (1.1) was derived based on a general equilibrium model that was both proposed and tested in a working paper, and which was subsequently published [22]. It is shown in $[14,21]$ that (1.1) possesses well defined viscosity solutions [4]. To this end, a high degree of smoothness for the coefficients and the prepayment function $h(\mathbf{x}, t)$ is assumed. Also the quadratic non-linearity in (1.1) poses a hindrance to regularity and convergence arguments, and thus requires the authors in $[14,21]$ to employ viscosity techniques.

Our first aim in [12] was to circumvent these hindrances. Note that a steady decline of interest rates was observed in the U.S markets, following the recession of 2008; see Figure 1.1. This was one of the sharpest declines in the markets history $[19]$.

We decided to consider a simplification of this economic scenario based on the following assumptions:

(1) The price $u$ depends on four economic factors and time,

$$
u(\mathbf{x}, t)=u\left(x_{1}, x_{2}, x_{3}, x_{4}, t\right) .
$$

(2) The prepayment function depends only on interest rate and time,

$$
S(\mathbf{x}, t)=S\left(x_{4}, t\right) .
$$

We want to consider a economic scenario of decreasing interest rates,

$$
x_{4} \searrow 0
$$

\footnotetext{
${ }^{1}$ Also $M B(t)$ is the remaining principal on a mortgage at time $t$. When there are no prepayments we have

$$
M B(t)=M B(0) \frac{e^{\tau^{\prime} T}-e^{\tau^{\prime} t}}{e^{\tau^{\prime} T}-1},
$$

where $\tau^{\prime}$ is the fixed rate paid by the mortgagor, while the investor receives $\tau<\tau^{\prime}$. We also assume $h(t)+\xi(t)>0$.
} 


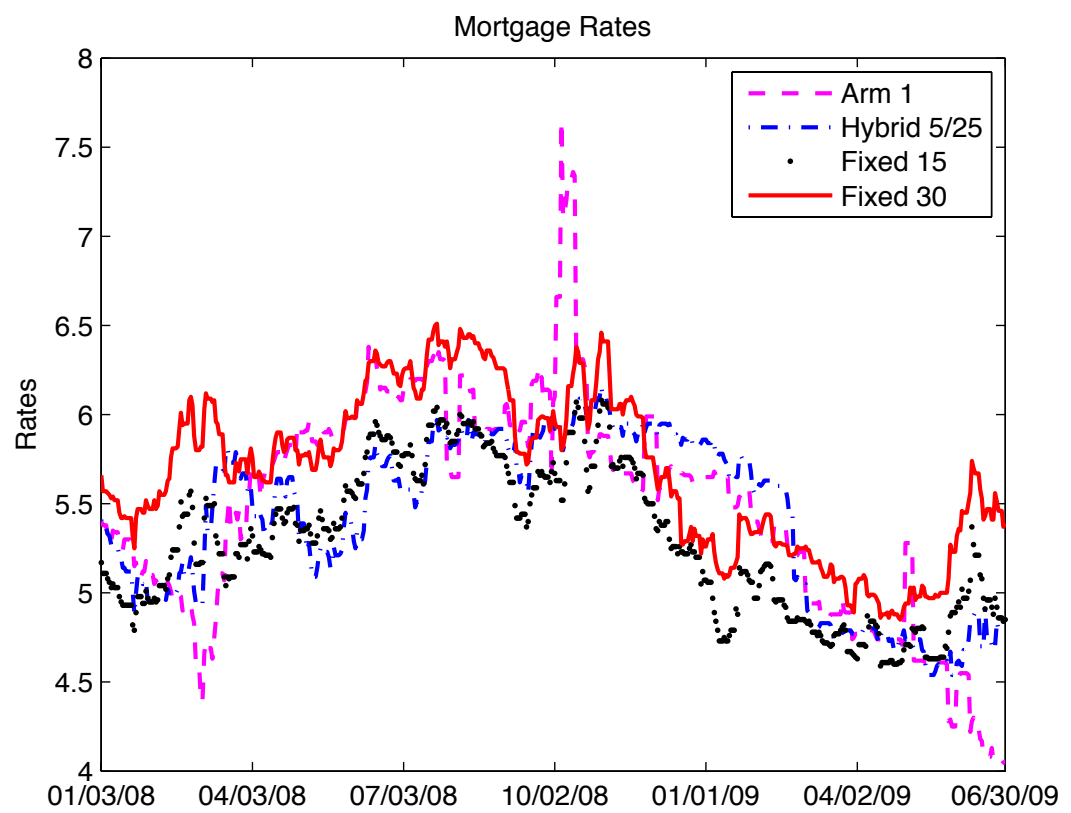

FIG. 1.1. Notice the steep fall in interest rates from October 2008- June 2009.

(3) We consider the limiting situation

$$
S\left(x_{4}, t\right)=\lim _{x_{4} \rightarrow 0} g(t) e^{-\beta_{4} x_{4}}=g(t)=S(t) .
$$

(4) We consider a cyclical economy, best mimicked via periodic boundary conditions.

The interest rate cannot hit 0 in reality, but our assumption is merely a construct to mimic the real economic scenario of sharply falling interest rates. This however greatly simplified (1.1), and enabled us to prove the following result in [12].

Lemma 1.1. The MBS Equation (1.1) can be reduced to a diffusion equation of the type

$$
\frac{\partial V}{\partial t}=\frac{1}{2} \Delta V+\tilde{K}(V, t) \quad \text { in } \quad U, V=0 \quad \text { on } \partial U, V(\mathbf{x}, 0)=V_{0}(\mathbf{x})
$$

where

$$
\begin{aligned}
\tilde{K}(V, t) & =(1-2 C \rho) F(t)\left(e^{\left(\frac{1}{2 C \rho-1}\right) t}\right)(V+l)^{\left(\frac{2 C \rho}{2 C \rho-1}\right)}-\frac{\partial l}{\partial t}+\frac{1}{2} \Delta l, \\
F(t) & =\tau h(t)+r \xi(t)+h^{\prime}(t)+\xi^{\prime}(t), \\
l(\mathbf{x}, t) & =(h(t)+\xi(t))^{(1-2 C \rho)} e^{-\left(\left((2 C \rho-1) r-\frac{(\mu)^{2}}{2}\right) t+\mu \mathbf{x}\right) .}
\end{aligned}
$$

We will briefly review the relationship between the original model (1.1) and our reduced model (1.7). Essentially (1.7) is derived after a series of transformations are 
performed on the variable $u$ in (1.1). We refer the reader to [12] for a full derivation. The gist of it is that $u$ and $V$ are related by the following relation:

$$
u(x, t)+h(t)+\xi(t)=(V(x, t)+l(x, t))^{\frac{1}{1-2 C_{\rho}}} e^{s(x, t)} .
$$

In particular, since $u(x, t)+h(t)>0$ by financial constraint, that is positive price of an MBS $[13,21,22]$, we have that

$$
(V(x, t)+l(x, t)) \geq \xi(t)^{1-2 C \rho} e^{-s(x, t)} \geq \delta_{1}>0,
$$

where $\delta_{1}$ depends on the parameters appearing in $\xi$ and $s$. This is important for the Lipschitz continuity of $K(V)$ to follow. On a financial level, this is saying that $V$ is essentially discounted from $u$, according to the scaling again given by the parameters appearing in $s$. Here $U$ is a bounded domain in $\mathbb{R}^{3}$.

We next briefly review the relevant literature. Since the seminal work of Black and Scholes [1], much importance has been given to pricing of derivative securities as partial differential equations. Thus there is a fair body of recent literature on the pricing of MBS via PDE methods. The work of Goncharov [8] considers flaws in traditional option based approaches. He derives a PDE for the pricing of MBS via a reduced option based or MRB approach. Goncharov derives the PDE as a solution of a fixed point problem to an integral equation. Brianni and Papi in [14], derived a non linear PDE, for the price of a mortgage backed security, via Malliavin calculus techniques. They claim that theirs is a non linear relation between the price of an MBS, interest rates, and prepayments. Various regularity results are presented for this equation in [21]. The recent work of Sharp [15] is yet another PDE approach to mortgage pricing. Sharp assumes that housing prices and interest rates are the only source of uncertainty in the economic environment, and then uses perturbation methods, common in the fluid dynamics literature, to derive an asset valuation PDE for a mortgage. Sharp also numerically solves his model to value both fixed and adjustable rate mortgages.

The organisation of the current manuscript is as follows. We first prove that the reduced model possesses a weak solution under different boundary conditions than those prescribed in [12], namely periodic boundary conditions. This is essential for the implementation of our numerical method. Periodic boundary conditions are used quite often in the financial literature [23, 24]; they mimic our assumption of a cyclical economy. This is seen quite often in standard business cycle models [25]. Since the non linear term is Lipschitz continuous, the existence proof for a weak solution falls within scope of classical results, say via Galerkin truncation. We use a fixed point method, and thus present few details. We then show that under additional regularity assumptions on the initial data, the model further possesses a mild solution. Next we prove that this mild solution is actually a strong solution. We also numerically solve this model via a Fourier spectral method, and compare our solution to actual prices realised in the MBS market. We note that our reduced model captures a number of market trends observed immediately following the recession.

\section{Weak solution}

In this section we will show the existence of a weak solution to the reduced MBS model with prescribed periodic boundary conditions. In all estimates made henceforth $C$ is a generic constant that can change in its value from line to line, and sometimes within the same line if so required.

We first state the following result: 
Lemma 2.1. Consider the reduced MBS model

$$
\begin{aligned}
& \frac{\partial V(\mathbf{x}, t)}{\partial t}=\frac{1}{2} \Delta V+\tilde{K}(V, t) \quad \text { in } U, \\
& V(0, y, z)=V(L, y, z), \quad V(x, 0, z)=V(x, L, z), \quad V(x, y, 0)=V(x, y, L), \\
& \frac{V(0, y, z)}{\partial x}=\frac{V(L, y, z)}{\partial x}, \quad \frac{V(x, 0, z)}{\partial y}=\frac{V(x, L, z)}{\partial y}, \quad \frac{V(x, y, 0)}{\partial z}=\frac{V(x, y, L)}{\partial z} \text {, } \\
& V(\mathbf{x}, 0)=V_{0}(\mathbf{x}) \text {. }
\end{aligned}
$$

For $C \rho<1 / 2$ there exists a unique weak solution $V$ to the above equation, with

$$
V \in L^{2}\left(0, T ; H^{1}(U)\right) \cap L^{\infty}\left(0, T ; L^{2}(U)\right)
$$

and

$$
\frac{\partial V}{\partial t} \in L^{2}\left(0, T ; H^{-1}(U)\right)
$$

In order to proceed we will derive certain properties that $\tilde{K}(V, t)$ satisfies. The first of these is Lipschitz continuity in $V$, in the $L^{2}$ norm. This was derived via the following Lemma in [12]:

Lemma 2.2. Consider the function

$$
\tilde{K}(V, t)=(1-2 C \rho) F(t)\left(e^{\left(\frac{1}{2 C \rho-1}\right) t}\right)(V+l)^{\left(\frac{2 C \rho}{2 C \rho-1}\right)}-\frac{\partial l}{\partial t}+\frac{1}{2} \Delta l .
$$

If $C \rho<1 / 2$ then $\tilde{K}(V, t)$ is Lipschitz continuous in the $L^{2}$ norm with respect to the variable $V$.

The proof follows due to the uniform boundedness of the derivative of $K$ with respect to $V$. Essentially,

$$
\begin{aligned}
& \frac{d}{d V}(1-2 C \rho) F(t)\left(e^{\left(\frac{1}{2 C \rho-1}\right) t}\right)(V+l)\left(\frac{2 C \rho}{2 C \rho-1}\right)-\frac{\partial l}{\partial t}+\frac{1}{2} \Delta l \\
\leq & C\left(\frac{1}{2 C \rho-1}\right)(V+l)^{\frac{1}{2 C \rho-1}} \\
\leq & C\left(\frac{1}{2 C \rho-1}\right) C\left(\frac{1}{\delta_{1}}\right)^{\frac{1}{2 C \rho-1}} .
\end{aligned}
$$

This follows due to (1.9). Thus we have

$$
\left|\frac{d}{d V} \tilde{K}(V)\right|_{\infty} \leq\left(\frac{C}{\delta_{1}}\right)^{\frac{1}{2 C \rho-1}},
$$

and the Lipschitz continuity of $\tilde{K}$ in $V$ follows.

The next property, an a priori estimate in $L^{2}\left(0, T ; L^{2}(U)\right)$ of $\tilde{K}$, is a trivial consequence of the Lipschitz continuity. This is stated via the following Lemma.

LEMMA 2.3. The function $\tilde{K}(V, t)$ satisfies the following a priori estimate for $C \rho<\frac{1}{4}$ :

$$
|\tilde{K}(V, t)|_{L^{2}\left(0, T ; L^{2}(U)\right)} \leq C .
$$


The constant $C$ depends only on the $L^{2}$ norm of the initial data. If $1 / 4<C \rho<1 / 2$ then we require the initial data to be in $L^{\alpha}$, where

$$
\alpha=\frac{2 C \rho}{1-2 C \rho}>2 .
$$

\subsection{Existence of a solution.}

Proof. Since the nonlinear term in the equation is Lipschitz, the existence of weak solution follows via classical truncation methods; see [6]. However we present a variant of a classical proof via a fixed point method. We consider the space

$$
X=C\left([0, T] ; L^{2}(U)\right)
$$

equipped with a supremum type norm

$$
|V|=\max _{t \leq 0 \leq T}|V(t)|_{L^{2}(U)} .
$$

On this space we define an appropriate operator $A$,

$$
A[V]=g .
$$

The idea is show that $A$ induces a contraction under the dynamics of the reduced MBS model, for $T$ chosen small enough. For a given $V \in X$, we set

$$
H(t)=\tilde{K}(V, t),
$$

and proceed via the standard energy method technique. We insert $\tilde{K}(V, t)$ for $H(t)$ where appropriate, as this is feasible via Lemma 2.3.

We consider two solutions $g$ and $\tilde{g}$ with the same period and initial conditions. Via the definition of $A$ we have

$$
A[V]=g, A[\tilde{V}]=\tilde{g}
$$

Now $g-\tilde{g}$ satisfies

$$
\begin{gathered}
\frac{\partial(g(\mathbf{x}, t)-\tilde{g}(\mathbf{x}, t))}{\partial t}=\frac{1}{2} \Delta(g(\mathbf{x}, t)-\tilde{g}(\mathbf{x}, t))+H(t)-\tilde{H}(t) \quad \text { in } U \\
g(\mathbf{x}, t)-\tilde{g}(\mathbf{x}, t)=0 \quad \text { on } \partial U \\
g(\mathbf{x}, 0)-\tilde{g}(\mathbf{x}, 0)=0 .
\end{gathered}
$$

We multiply (2.2) by $g-\tilde{g}$ and integrate by parts over $U$, use Cauchy with epsilon, and obtain

$$
\begin{aligned}
\frac{d}{d t}|g-\tilde{g}|_{2}^{2}+2|g-\tilde{g}|_{2}^{2} & \leq C \epsilon|g-\tilde{g}|_{2}^{2}+\frac{1}{\epsilon}|H-\tilde{H}|_{2}^{2} \\
& \leq C \epsilon|g-\tilde{g}|_{2}^{2}+\frac{C}{\epsilon}|V-\tilde{V}|_{2}^{2} .
\end{aligned}
$$

This follows as $\tilde{K}(V, t)$ is Lipschitz in $\mathrm{V}$. We choose $\epsilon$ such that $2>C \epsilon$, to yield

$$
\frac{d}{d t}|g-\tilde{g}|_{2}^{2}+(2-C \epsilon)|g-\tilde{g}|_{2}^{2} \leq C|V-\tilde{V}|_{2}^{2} .
$$


As $(2-C \epsilon)|g-\tilde{g}|_{2}^{2}>0$ we obtain

$$
\frac{d}{d t}|g-\tilde{g}|_{2}^{2} \leq C|V-\tilde{V}|_{2}^{2} .
$$

Now via the definition of $A$, we obtain $g=A[V]$; therefore,

$$
\frac{d}{d t}|A[V]-A[\tilde{V}]|_{2}^{2} \leq C|V-\tilde{V}|_{2}^{2}
$$

Integration in the time interval $[0, T]$ yields

$$
|A[V]-A[\tilde{V}]|_{2} \leq C \int_{0}^{T}|V-\tilde{V}|_{2}^{2} \leq(C T)^{\frac{1}{2}}|V-\tilde{V}|_{2} .
$$

Choosing $T$ such that

$$
(C T)^{1 / 2} \leq \gamma \leq 1
$$

implies that for any $t<T_{1}=1 / C$,

$$
|A[V]-A[\tilde{V}]|_{2} \leq \gamma|V-\tilde{V}|_{2}, \quad \gamma<1 .
$$

Thus for a given $V \in X, A$ generates a contraction on the time interval $\left[0, T_{1}\right]$. Thus via a direct application of the Banach fixed point theorem [3], the operator A must have a fixed point $V^{*}$. Thus

$$
A\left[V^{*}\right]=V^{*}
$$

However via the definition of $A, A[V]=g$, so

$$
A\left[V^{*}\right]=V^{*}=g .
$$

This implies the existence of a $V^{*}$ which is also a solution to the reduced model [6]. The solution is valid on the short time interval $\left[0, T_{1}\right]$. We can now repeat the above argument to extend the solution to say $\left[T_{1}, 2 T_{1}\right]$ and eventually cover $[0, T]$. We can show that the weak solution also belongs to $L^{\infty}\left(0, T ; L^{2}(U)\right)$ via standard Galerkin truncation for $V$.

This completes the proof.

\section{Mild and strong solution}

3.1. Mild solution. In this section we establish that the reduced PDE model actually possesses mild solution, which in turn is a strong solution. In order to demonstrate this we will first recap the essential theory. This is shown via additional requirements on the non-linearity. We follow the approach of [18]. We first recall the requisite methodology. Consider the general non linear evolution equation

$$
\frac{\partial u}{\partial t}+A u=F(u)
$$

Definition 3.1 (Standing Hypothesis A). Let A be a positive sectorial operator on a Banach space $W$ with associated analytic semigroup $e^{-A t}$. Let $V^{2 \alpha}$ be the family of interpolation spaces generated by the fractional powers of A, where 
$V^{2 \alpha}=D\left(A^{\alpha}\right)$, for $\alpha \geq 0$. Let $\left\|A^{\alpha} u\right\|=\left\|A^{\alpha} u\right\|_{W}=\|u\|_{V^{2 \alpha}}=\|u\|_{2 \alpha}$ denote the norm on $V^{2 \alpha}$.

Definition 3.2 (The Space $C_{\text {Lip }}$ ). Let $V$ and $W$ denote two Banach spaces and let $C_{\text {Lip }}=C_{\text {Lip }}\left(V \times \mathbb{R}^{+} ; W\right)$ denote the collection of all continuous functions $F$ : $V \times \mathbb{R}^{+} \rightarrow W$ with the property that for any bounded set $B$ in $V$ there exists constants $K_{0}=K_{0}(B)$ and $K_{1}=K_{1}(B)$, such that

$$
\|F(v, t)\|_{W} \leq K_{0}, \text { for all } v \in B \text { and } t \in \mathbb{R}^{+},
$$

and for all $v_{1} \in B$ and $t \in \mathbb{R}^{+}$

$$
\left\|F\left(v_{1}, t\right)-F\left(v_{2}, t\right)\right\|_{W} \leq K_{1}\left\|v_{1}-v_{2}\right\|_{V} .
$$

We now state a standard Lemma for mild solutions.

Lemma 3.3 (Sell, You). Consider the non-linear evolutionary equation

$$
\frac{\partial u}{\partial t}+A u=F(u, t), u\left(t_{0}\right)=u_{0} \in W,
$$

Let the standing Hypothesis $A$ be satisfied and let

$$
F \in C_{\text {Lip }}=C_{\text {Lip }}\left(V^{2 \beta} \times \mathbb{R}^{+} ; W\right),
$$

where $0 \leq \beta<1$. Then for every $u_{0} \in V^{2 \beta}$, there is a $T>0$ such that the above evolutionary equation has a unique mild solution $u=u(t)$ in $V^{2 \beta}$ with

$$
u \in C\left[0, T ; V^{2 \beta}\right) \cap C_{l o c}^{0, \theta_{1}}\left(0, T ; V^{2 \alpha}\right) \cap C_{l o c}^{0, \theta}\left(0, T ; V^{2 r}\right),
$$

for all $\alpha$ and $r$ with $0 \leq \alpha \leq \beta$ and $0 \leq r<1$, where $\theta_{1}>0$ and $\theta>0$.

REMARK 3.1. $C^{k, \lambda}$ is taken to be the standard function space of Hölder continuous functions with Hölder exponent $\lambda$.

We now state the following result:

Lemma 3.4. Consider the reduced MBS model described in Theorem 2.1. For initial data in $H^{1}(U)$, there is a $T>0$ such that the model has a unique mild solution in $H^{1}(U)$. Furthermore the solution has the following regularity

$$
V \in C\left[0, T ; H^{1}(U)\right) \cap C_{l o c}^{0, \theta_{1}}\left(0, T ; H^{1}(U)\right) \cap C_{l o c}^{0, \theta}\left(0, T ; H^{2-\delta}(U)\right),
$$

where $0<\theta_{1}, 0<\theta, 0<\delta<<1$.

Proof. In our case the operator $A=-\Delta$. Thus the standing hypothesis A is satisfied; see [18]. Furthermore, in our setting $W=L^{2}(U)$. We choose $\alpha=\beta=\frac{1}{2}$, thus $V^{2 \alpha}=V^{2 \beta}=H^{1}(U)$. Also the Lipschitz continuity of $K(V, t)$ in the variable $\mathrm{V}$ follows from Lemma 2.2, thus via compact Sobolev embedding of

$$
H^{1}(U) \hookrightarrow L^{2}(U)
$$

we have

$$
\left\|\tilde{K}\left(v_{1}, t\right)-\tilde{K}\left(v_{2}, t\right)\right\|_{2} \leq K_{1}\left\|v_{1}-v_{2}\right\|_{2} \leq C K_{1}\left\|v_{1}-v_{2}\right\|_{H^{1}(U)} .
$$

We can now use the above estimate via our above choice of spaces, to show that the non-linearity in question, $\tilde{K}(V, t)$, is a bounded map from

$$
H^{1}(U) \mapsto C_{L i p}\left(H^{1}(U) \times \mathbb{R}^{+} ; L^{2}(U)\right) .
$$

Thus the result follows as a direct application of Lemma 3.3. 
3.2. Strong solution. Before we tackle the case of the strong solution, further theory must be developed.

Definition 3.5 (The Space $C_{\text {Lip; } ;}$ ). For $\theta$ satisfying $0<\theta \leq 1$, we define $C_{\text {Lip; } \theta}\left(V \times \mathbb{R}^{+} ; W\right)$ to denote the collection of all continuous functions $F \in C_{\text {Lip }}(V \times$ $\left.\mathbb{R}^{+} ; W\right)$ with the property that for any bounded set $B$ in $V$ and every compact set $J$ in $R^{+}$, there exists a constant $K_{2}=K_{2}(B, J)$ such that

$$
\left\|F\left(w_{1}, t_{1}\right)-F\left(w_{2}, t_{2}\right)\right\| \leq K_{2}\left(\left\|w_{1}-w_{2}\right\|+\left|t_{1}-t_{2}\right|^{\theta}\right) \text { for all } v \in B \text { and } t \in \mathbb{R}^{+} .
$$

We now state the following standard Lemma.

Lemma 3.6. Consider the non-linear evolutionary equation

$$
\frac{\partial u}{\partial t}+A u=F(u, t), u\left(t_{0}\right)=u_{0} \in W
$$

Let the standing Hypothesis $A$ be satisfied and let

$$
F \in C_{L i p ; \theta}=C_{L i p ; \theta}\left(V^{2 \beta} \times \mathbb{R}^{+} ; W\right),
$$

where $0 \leq \beta<1$ and $0<\theta \leq 1$. Then for every $u_{0} \in V^{2 \beta}$, there is a $T>0$ such that the mild solution of the above evolutionary equation in $V^{2 \beta}$ is a unique strong solution in $V^{2 \beta}$ with

$$
u \in C\left[0, T ; V^{2 \alpha}\right) \cap C_{l o c}^{0,1-r}\left(0, T ; V^{2 r}\right) \cap C(0, T ; D(A)),
$$

for all $\alpha$ and $r$ with $0 \leq \alpha \leq \beta$ and $0 \leq r<1$.

In order to prove such a result for our system of interest it is essential to make more apriori estimates. We begin with the following Lemma.

LEMma 3.7. $h(t)$ and $\xi(t)$ given by

$$
\begin{gathered}
h(t)=M B(t) e^{-\left(\frac{p \gamma(\gamma t)^{P}-1}{1+(\gamma t)^{P}}\right)} \\
r(t)=\delta(T-t), \quad \xi(t)=A_{0} e^{\int_{0}^{T-t} \delta(s) d s}
\end{gathered}
$$

are both Lipschitz functions in time, under the assumption that the interest rate process $\delta(t)$ is positive and bounded ${ }^{2}, \gamma>0, p>1$, and $h(t)+\xi(t)>0$.

\footnotetext{
${ }^{2}$ In deriving the reduced model we actually assume the interest rate process $\delta(t)$ is a constant. However the Lemma can be proven even if it is not, under feasible assumptions on its functional form. Also without loss of generality we assume $A_{0}>0$, for if not the result follows also follows by taking the supremum norm in the inequality for $\frac{d \xi}{d t}$ presented in the proof of the Lemma.
} 
Proof. We begin by estimating the time derivative of $h$,

$$
\begin{aligned}
& \left|\frac{d h}{d t}\right|_{\infty} \\
\leq & \left|e^{-\left(\frac{p \gamma(\gamma t)^{P-1}}{1+(\gamma t)^{P}}\right)}\right|_{\infty}\left|M B(t) \frac{d\left(\frac{p \gamma(\gamma t)^{P-1}}{1+(\gamma t)^{p}}\right)}{d t}+M B^{\prime}(t)\right|_{\infty} \\
\leq & \left|e^{-\left(\frac{p \gamma(\gamma t)^{P-1}}{1+(\gamma t)^{P}}\right)}\right|_{\infty}|M B(t)|_{\infty}\left|\frac{d\left(\left(\frac{p \gamma(\gamma t)^{P-1}}{1+(\gamma t)^{P}}\right)\right)}{d t}\right|_{\infty} \\
& +\left|e^{-\left(\frac{p \gamma(\gamma t)}{1+(\gamma t)^{P}}\right)}\right|_{\infty}\left|M B^{\prime}(t)\right|_{\infty} \\
\leq & C .
\end{aligned}
$$

This follows as for $t>0, \frac{p \gamma(\gamma t)^{P-1}}{1+(\gamma t)^{p}}>0$, and for $t>0, M B(t)$ and $\left(\frac{p \gamma(\gamma t)^{P-1}}{1+(\gamma t)^{p}}\right)$ have bounded derivative from their functional forms.

Taking the derivative of $\xi$ yields

$$
\frac{d \xi}{d t}=-\delta(T-t) \xi(t)
$$

Since $\delta(t)$ is a continuous, bounded positive function in $[0, T]$, it attains a minimum in $[0, T]$, say $\min (\delta(t))_{t \in[0, T]}=K$, and a maximum in $[0, T]$, say $\max (\delta(t))_{t \in[0, T]}=K_{1}$. Thus we have $K \leq \delta(T-t) \leq K_{1}$. This implies

$$
-K_{1} \xi(t) \leq \frac{d \xi}{d t} \leq-K \xi(t)
$$

Hence via Gronwall's inequality

$$
\xi(t) \leq \xi(0) e^{-K T} \leq A_{0} e^{K_{1} T} e^{-K T} \leq C .
$$

Thus it follows that

$$
\left|\frac{d \xi}{d t}\right|_{\infty} \leq\left|-K_{1} \xi(t)\right|_{\infty} \leq K_{1}|\xi(t)|_{\infty} \leq C
$$

This is seen due to the assumption on the rate process. Thus the result follows, as derivatives of $h(t)$ and $\xi(t)$ are bounded uniformly in time.

REMARK 3.2. It follows via an application of the chain rule that $(h(t)+\xi(t))^{(1-2 C \rho)}$, $(h(t)+\xi(t))^{(-2 C \rho)}, h^{\prime}(t)$, and $\xi^{\prime}(t)$ also have bounded derivatives in time, and hence are Lipschitz functions in time.

We now state the following result.

Theorem 3.8. Consider the reduced MBS model described in Theorem 2.1. For initial data in $H^{1}(U)$, there is a $T>0$ such that the model has a unique strong solution in $H^{1}(U)$. Furthermore the solution has the following regularity:

$$
V \in C\left[0, T ; H^{1}(U)\right) \cap C_{l o c}^{0, \frac{1}{2}}\left(0, T ; H^{1}(U)\right) \cap C_{l o c}^{0,1}\left(0, T ; H^{2-\delta}(U)\right),
$$


where $0<\delta<<1$.

Proof. First note that

$$
\begin{gathered}
\Delta l=\mu^{2}(h(t)+\xi(t))^{(1-2 C \rho)} e^{-\left(\left((2 C \rho-1) r-\frac{(\mu)^{2}}{2}\right) t+\mu \mathbf{x}\right)}, \\
\frac{\partial l}{\partial t}=-\left((2 C \rho-1) r-\frac{(\mu)^{2}}{2}\right)(h(t)+\xi(t))^{(1-2 C \rho)} e^{-\left(\left((2 C \rho-1) r-\frac{(\mu)^{2}}{2}\right) t+\mu \mathbf{x}\right)} \\
+(1-2 C \rho)(h(t)+\xi(t))^{(-2 C \rho)}\left(h^{\prime}(t)+\xi^{\prime}(t)\right) e^{-\left(\left((2 C \rho-1) r-\frac{(\mu)^{2}}{2}\right) t+\mu \mathbf{x}\right)},
\end{gathered}
$$

and

$$
\begin{aligned}
\tilde{K}(V, t)= & (1-2 C \rho) F(t)\left(e^{\left(\frac{1}{2 C \rho-1}\right) t}\right)(V+l)^{\left(\frac{2 C \rho}{2 C \rho-1}\right)} \\
& +\left((2 C \rho-1) r-\frac{(\mu)^{2}}{2}\right)(h(t)+\xi(t))^{(1-2 C \rho)} e^{-\left(\left((2 C \rho-1) r-\frac{(\mu)^{2}}{2}\right) t+\mu \mathbf{x}\right)} \\
& +(1-2 C \rho)(h(t)+\xi(t))^{(-2 C \rho)}\left(h^{\prime}(t)+\xi^{\prime}(t)\right) e^{-\left(\left((2 C \rho-1) r-\frac{(\mu)^{2}}{2}\right) t+\mu \mathbf{x}\right)} \\
& +\mu^{2}(h(t)+\xi(t))^{(1-2 C \rho)} e^{-\left(\left((2 C \rho-1) r-\frac{(\mu)^{2}}{2}\right) t+\mu \mathbf{x}\right)} .
\end{aligned}
$$

Hence we can make the following estimate

$$
\begin{aligned}
\left|\tilde{K}\left(V_{1}, t_{1}\right)-\tilde{K}\left(V_{2}, t_{2}\right)\right|_{2} \leq & (1-2 C \rho) F\left(t_{1}\right)\left(e^{\left(\frac{1}{2 C \rho-1}\right) t_{1}}\right)\left(V_{1}+l\right)^{\left(\frac{2 C \rho}{2 C \rho-1}\right)} \\
& -(1-2 C \rho) F\left(t_{2}\right)\left(e^{\left(\frac{1}{2 C \rho-1}\right) t_{2}}\right)\left(V_{2}+l\right)^{\left(\frac{2 C \rho}{2 C \rho-1}\right)} \\
& +\left|\frac{\partial l\left(t_{1}\right)}{\partial t}-\frac{\partial l\left(t_{2}\right)}{\partial t}\right|_{2}+\left|\Delta l\left(t_{1}\right)-\Delta l\left(t_{2}\right)\right|_{2} \\
\leq & C\left|V_{1}-V_{2}\right|_{2}+\left|\frac{\partial l\left(t_{1}\right)}{\partial t}-\frac{\partial l\left(t_{2}\right)}{\partial t}\right|_{2}+\left|\Delta l\left(t_{1}\right)-\Delta l\left(t_{2}\right)\right|_{2} .
\end{aligned}
$$

This follows as the Lipschitz continuity of $F(t)\left(e^{\left(\frac{1}{2 C \rho-1}\right) t}\right)\left(V_{1}+l\right)^{\left(\frac{2 C \rho}{2 C \rho-1}\right)}$ in $V$ is immediate from Lemma 2.2. Hence we now estimate

$$
\begin{aligned}
& \left|\frac{\partial l\left(t_{1}\right)}{\partial t}-\frac{\partial l\left(t_{2}\right)}{\partial t}\right|_{2} \\
\leq & \left|\left((2 C \rho-1) r-\frac{(\mu)^{2}}{2}\right) e^{-\left((2 C \rho-1) r-\frac{(\mu)^{2}}{2}\right) t_{1}}\right|_{\infty} \\
& \times\left|\left(h\left(t_{1}\right)+\xi\left(t_{1}\right)\right)^{(1-2 C \rho)}-C_{0}\left(h\left(t_{2}\right)+\xi\left(t_{2}\right)\right)^{(1-2 C \rho)}\right|_{2} \\
& +\left|\left((2 C \rho-1) r-\frac{(\mu)^{2}}{2}\right)\right|_{\infty}
\end{aligned}
$$


1044 STRONG SOLUTION OF A CLASS OF PARTIAL DIFFERENTIAL EQUATIONS

$$
\begin{aligned}
& \quad\left|\left(h\left(t_{2}\right)+\xi\left(t_{2}\right)\right)^{(1-2 C \rho)}\right|_{\infty}\left|e^{-\left((2 C \rho-1) r-\frac{(\mu)^{2}}{2}\right) t_{2}}-e^{-\left((2 C \rho-1) r-\frac{(\mu)^{2}}{2}\right) t_{1}}\right|_{2} \\
& +\left|\left(h^{\prime}\left(t_{1}\right)+\xi^{\prime}\left(t_{1}\right)\right) e^{-\left((2 C \rho-1) r-\frac{(\mu)^{2}}{2}\right) t_{1}}\right|_{\infty} \\
& \times\left|\left(h\left(t_{1}\right)+\xi\left(t_{1}\right)\right)^{(-2 C \rho)}-\left(h\left(t_{2}\right)+\xi\left(t_{2}\right)\right)^{(-2 C \rho)}\right|_{2} \\
& +\left|\left(h\left(t_{2}\right)+\xi\left(t_{2}\right)\right)^{(-2 C \rho)}\right|_{\infty} \\
& \times\left|\left(h^{\prime}\left(t_{1}\right)+\xi^{\prime}\left(t_{1}\right)\right) e^{-\left((2 C \rho-1) r-\frac{(\mu)^{2}}{2}\right) t_{1}}-\left(h^{\prime}\left(t_{2}\right)+\xi^{\prime}\left(t_{2}\right)\right) e^{-\left((2 C \rho-1) r-\frac{(\mu)^{2}}{2}\right) t_{2}}\right|_{2} \\
& \leq C_{1}\left|\left(h\left(t_{1}\right)+\xi\left(t_{1}\right)\right)^{(1-2 C \rho)}-\left(h\left(t_{2}\right)+\xi\left(t_{2}\right)\right)^{(1-2 C \rho)}\right|_{2}+C_{2} \\
& +C_{3}\left|\left(h\left(t_{1}\right)+\xi\left(t_{1}\right)\right)^{(-2 C \rho)}-\left(h\left(t_{2}\right)+\xi\left(t_{2}\right)\right)^{(-2 C \rho)}\right|_{2}+C_{4} \\
& \leq
\end{aligned}
$$

Note we assume $C_{0}>1$, for if $C_{0}<1$ then the estimate is of the form

$$
\left|\frac{\partial l\left(t_{1}\right)}{\partial t}-\frac{\partial l\left(t_{2}\right)}{\partial t}\right|_{2} \leq C_{5}\left|t_{1}-t_{2}\right|_{2}+C_{7}
$$

where

$$
C_{7}=\left|\left((2 C \rho-1) r-\frac{(\mu)^{2}}{2}\right) e^{-\left((2 C \rho-1) r-\frac{(\mu)^{2}}{2}\right) t_{1}}\right|_{\infty}\left|\left(1-C_{0}\right)\left(h\left(t_{1}\right)+\xi\left(t_{1}\right)\right)^{(1-2 C \rho)}\right|_{2} .
$$

We now estimate

$$
\begin{aligned}
& \left|\Delta l\left(t_{1}\right)-\Delta l\left(t_{2}\right)\right|_{2} \\
\leq & C\left|e^{-\left((2 C \rho-1) r-\frac{(\mu)^{2}}{2}\right) t_{1}}\right|_{\infty}\left|\left(h\left(t_{1}\right)+\xi\left(t_{1}\right)\right)^{(1-2 C \rho)}-\left(h\left(t_{2}\right)+\xi\left(t_{2}\right)\right)^{(1-2 C \rho)}\right|_{2} \\
& +\left|\left(h\left(t_{2}\right)+\xi\left(t_{2}\right)\right)^{(1-2 C \rho)}\right|_{\infty}\left|e^{-\left((2 C \rho-1) r-\frac{(\mu)^{2}}{2}\right) t_{1}}-e^{-\left((2 C \rho-1) r-\frac{(\mu)^{2}}{2}\right) t_{2}}\right|_{2} \\
\leq & C_{8}\left|t_{1}-t_{2}\right|+C_{9} .
\end{aligned}
$$

These estimates follow from Lemma 3.7. Therefore from the above we obtain

$$
\begin{aligned}
& \left|\tilde{K}\left(V_{1}, t_{1}\right)-\tilde{K}\left(V_{2}, t_{2}\right)\right|_{2} \\
\leq & C\left|V_{1}-V_{2}\right|_{2}+C_{5}\left|t_{1}-t_{2}\right|_{2}+C_{8}\left|t_{1}-t_{2}\right|_{2}+C_{6}+C_{9} \\
\leq & C_{10}\left|V_{1}-V_{2}\right|_{2}+C_{11}\left|t_{1}-t_{2}\right|_{2} .
\end{aligned}
$$

In our case the operator $A=-\Delta$. Thus the standing hypothesis $\mathrm{A}$ is satisfied; see [18]. Furthermore, in our setting $W=L^{2}(U)$. We choose $\alpha=\beta=\frac{1}{2}$, thus $V^{2 \alpha}=V^{2 \beta}=$ 
$H^{1}(U)$. W can now use the above estimates along with Lemma 3.6 to prove Theorem 3.8 , via our above choice of spaces. Essentially we show that the non-linearity in question, $\tilde{K}(V, t)$, is a bounded map from

$$
H^{1}(U) \mapsto C_{L i p, 1}\left(H^{1}(U) \times \mathbb{R}^{+} ; L^{2}(U)\right) .
$$

\section{Numerical solution and calibration with market data}

Our goal in this section is to describe how we numerically solve the reduced MBS model, derived in 2.1, in the 1-d case. Essentially, finding a solution to the reduced model is equivalent to solving (4.1), which is more tractable numerically. Thus we consider the following non linear boundary value problem, defined on a periodic domain:

$$
\begin{aligned}
w_{t}-\frac{1}{2} w_{x x}-\mu w_{x}+(1-2 \rho) r w & =(1-2 \rho) F(t) w^{m}, \quad 0 \leq x \leq L, \\
w(0, t) & =w(L, t), \\
w_{x}(0, t) & =w_{x}(L, t), \\
w(x, 0) & =\left(P_{0}+\xi(t)\right)^{1-2 \rho},
\end{aligned}
$$

where $m=\frac{2 \rho}{2 \rho-1}$ and $L$ is the domain length. The solutions are obtained using a Fourier spectral approach. The merits of spectral accuracy vs. finite difference techniques can be found in [16]. To obtain the desired temporal accuracy, it is useful to make a few transformations first. Taking the Fourier transform of (4.1) with respect to $x$ leads to

$$
\hat{w}_{t}+c(k) \hat{w}-(1-2 \rho) F(t) \mathcal{F}\left\{w^{m}\right\}=0,
$$

where $c(k)=\left[k^{2} / 2-\mu i k+(1-2 \rho) r\right]$ and $k$ is the wavenumber, defined through

$$
\hat{w}(k, t)=\mathcal{F}\{w\}=\int_{-\infty}^{\infty} w(x, t) e^{-i k x} d x .
$$

The operator $\mathcal{F}$ denotes the Fourier transform from $w(x, t)$ to $\hat{w}(k, t)$, while $\mathcal{F}^{-1}$ denotes the inverse Fourier transform from $\hat{w}(k, t)$ to $w(x, t)$. These operators can be replaced with their discrete Fourier counterparts, without loss of generality, in (4.2). If we now multiply (4.2) by the integrating factor $e^{c(k) t}$, the linear component is solved exactly, leading to

$$
\frac{d}{d t}\left[e^{c(k) t} \hat{w}\right]-e^{c(k) t}(1-2 \rho) F(t) \mathcal{F}\left\{w^{m}\right\}=0 .
$$

Through a final change of variable, $W=e^{c(k) t} \hat{w}$, we are left with the simplified nonlinear initial value problem

$$
\begin{gathered}
W_{t}-e^{c(k) t}(1-2 \rho) F(t) \mathcal{F}\left\{\left[\mathcal{F}^{-1}\left\{e^{-c(k) t} W\right\}\right]^{m}\right\}=0, \\
W(k, 0)=\mathcal{F}\{w(x, 0)\} .
\end{gathered}
$$

The above equation is solved using the explicit $4^{t h}$ order Runge-Kutta scheme (RK4). The operations $\mathcal{F}$ and $\mathcal{F}^{-1}$ are acheived using the FFT (Fast Fourier Transform) library available in MATLAB ${ }^{T M}$, which utilizes Cooley and Tukey's algorithm to 
compute the discrete Fourier transform [3]. The algorithm requires a set of wave numbers, given by $k \in \frac{\pi}{L}[-N \ldots N]$, where $N$ is the number of Fourier modes. The original variable is recovered at each time increment through successive inverse transformations: $W \rightarrow \hat{w} \rightarrow w \rightarrow u$. The final change of variable is obtained through

$$
u(x, t)=w(x, t)^{\frac{1}{1-2 \rho}}-h(t)-\xi(t),
$$

where $h(t)$ is a given by 1.2 .

To maintain numerical stability, the time-step $(\Delta t)$, scaled by the maximum real eigenvalue of $(4.5)$, should lie between zero and $\approx-2.78$ for RK4 [2]. The elimination of the linear term in (4.2) reduces this maximum eigenvalue, effectively increasing the region of stability. However, due to the varying coefficient and nonlinearity in (4.5), the precise eigenvalues are exhaustive to obtain, especially when varying certain parameters. It is far easier to reduce $\Delta t$ until a solution has converged. For the results presented here, a converged solution is obtained for $\Delta t=0.0014$. We choose a domain length of $L=2000$ and $N=120$; these choices are arbitrary, since the solution $u$ is observed to be independent of $x$ for the prescribed boundary and initial conditions. This allows the point of measurement along the domain to be arbitrary. We next present plots of our numerical solution vs actual data from the MBS markets immediately following the recession of 2008. The MBS's that are used in below graphs are the passthrough $^{3}$ securities that are issued by The Federal National Mortgage Association, commonly known as Fannie Mae. We used four cusips that are issued in 2005 and 2007.

Table 4 presents the characteristics of each security as of September 2, 2008. The original WAC and the original WAM are the weighted average coupon and maturity of the pool at the issuance date, respectively. The WAC is the interest that is received by the issuer from the pool of mortgages. For example, the issuer of FNMA 2005 5.0 receives the original WAC of $5.56 \%\left(\tau^{\prime}\right)$ while paying $5.0 \%(\tau)$ of interest to investors of this particular security. The difference is kept by the issuer as servicing and guaranteeing fees. The original amortization is the number of coupon payments that an investor receives and is used to calculate the amortization schedule of the MBS. Current amortization is the number of coupon payments left as of September 2,2008 . The current factor is the remaining percentage balance of the pool since the issuance date.

\begin{tabular}{cccccc} 
MBS & $\begin{array}{c}\text { Original } \\
\text { WAC }\end{array}$ & $\begin{array}{c}\text { Original } \\
\text { Amortization }\end{array}$ & $\begin{array}{c}\text { Original } \\
\text { WAM }\end{array}$ & $\begin{array}{c}\text { Current } \\
\text { Amortization }\end{array}$ & $\begin{array}{c}\text { Current } \\
\text { Factor }\end{array}$ \\
\hline \hline & $(\%)$ & $(\mathbf{m o s})$ & $(\mathbf{m o s})$ & $(\mathbf{m o s})$ & $(\%)$ \\
FNMA 2005 5.0 & 5.56 & 360 & 348 & 320 & 70 \\
FNMA 2007 5.0 & 5.78 & 360 & 359 & 341 & 94 \\
FNMA 2007 5.0 & 5.59 & 360 & 339 & 342 & 88 \\
FNMA 2007 5.5 & 5.94 & 180 & 179 & 346 & 81
\end{tabular}

TABLE 4.1. MBS Characteristics As of September 2, 2008.

In each figure, we used parameter values of $M B(0)=100, \mu=0.5$, and $\delta=0.02$. These are specified apriori, and are common to each cusip. Along with these, the parameter set $\left(p, \gamma, \rho, A_{0}\right)$, given in Table 4.2 , is obtained by calibration of

\footnotetext{
${ }^{3} \mathrm{~A}$ passthrough security is a pool of mortgages that is sold as shares or participation certificates. When a mortgage is included in the pool that is used as collateral for a mortgage passthrough security, the mortgage is said to be securitized [7].
} 
the model by using realized prices of each security from September 2, 2008 and October 30, 2010. Also, original amortization $(T)$ and current amortization $(t=$ $T$-current amortization) values are used in the calculations. During the calibration MATLAB ${ }^{T M}$ 's fmincon function is utilized by setting the tolerance levels of the step size $(T o l X)$ and change in the function value (TolFun) to $10^{-8}$. We determine the lower boundary for $A_{0}$ as $-P_{0}$ to have nonnegative starting value if the discount rate is zero. The initial clean price is determined by using the September 2, 2008 value for each security. As a summary, we solve the following optimization problem:

$$
\min _{p, \gamma, \rho, A_{0}} \sum_{i=1}^{N} \frac{1}{N}\left\{(u+h)_{i}-\text { Market Price }_{i}\right\}^{2},
$$

where $i=1$ represents the day of September 3, 2008 and $i=N$ stands for October 30, 2010. Initial prices are given for each security in their corresponding figure. The smooth solid line that represents the model generated price captures the increasing trend of the market prices as well as the price drop in the first three months. The calibration results are presented next.

\begin{tabular}{crcccc} 
MBS & CUSIPS & $p$ & $\gamma$ & $\rho$ & $A_{0}$ \\
\hline \hline FNMA 2005 5.0 & $31402 \mathrm{RF} 87$ & 3.38 & 1.40 & 1.25 & -56.80 \\
FNMA 2007 5.0 & 31405 WXH3 & 0.70 & 1.18 & 1.25 & -54.60 \\
FNMA 2007 5.0 & 31410 F3P8 & 0.67 & 1.25 & 1.25 & -54.58 \\
FNMA 2007 5.5 & 31371 NHX6 & 2.15 & 2.58 & 1.25 & -54.18
\end{tabular}

TABle 4.2. MBS Model Parameters.

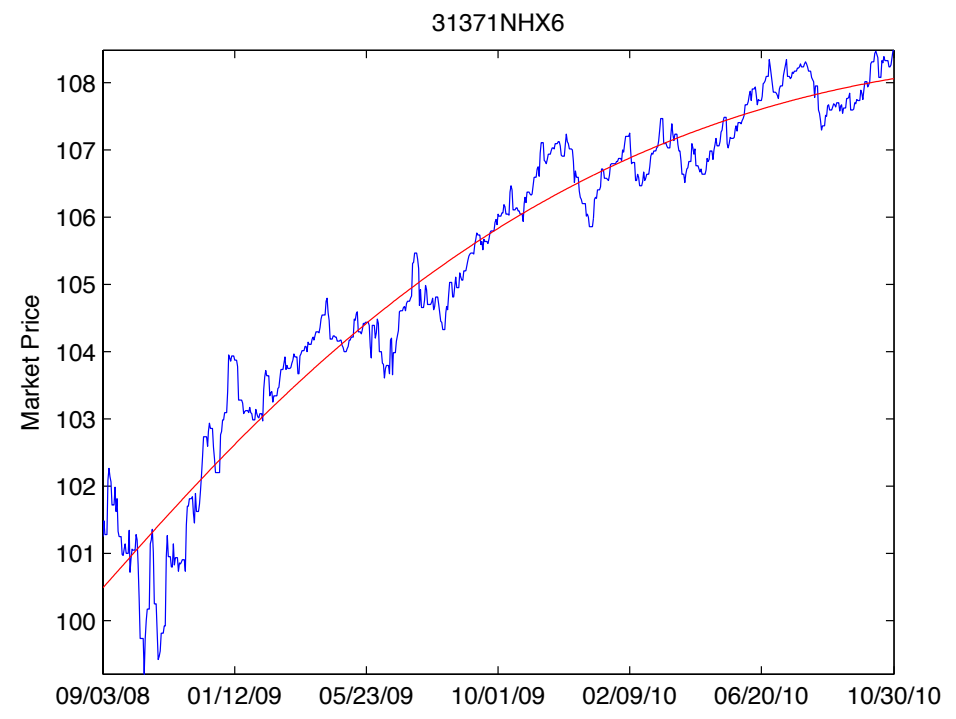

FIG. 4.1. Model Price v.s. Market Price: T=360 months, $P_{0}=101.16$. 


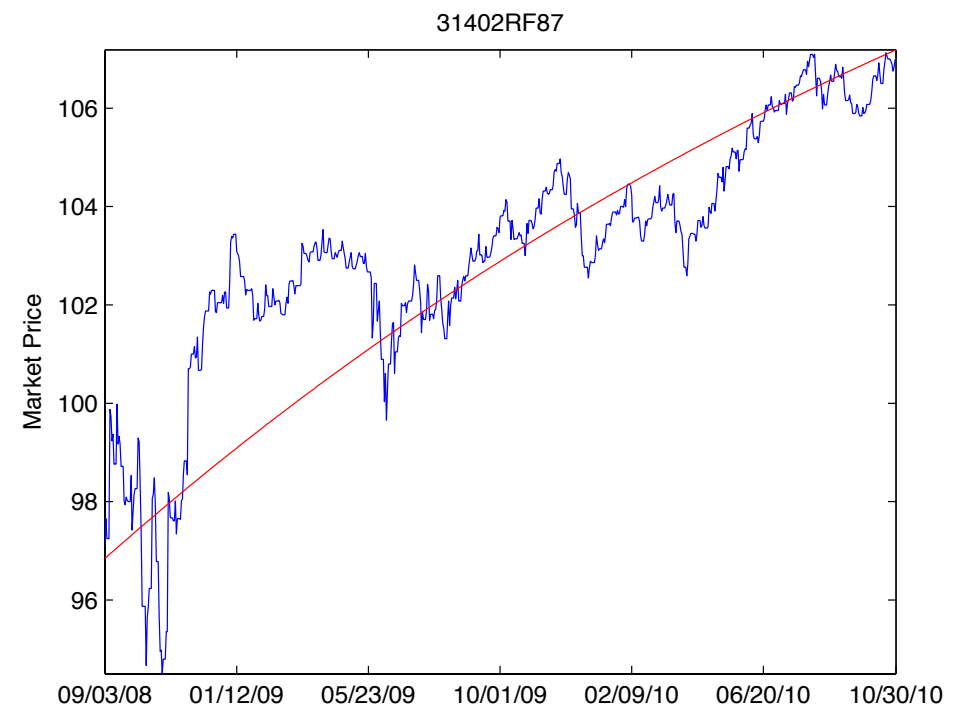

Fig. 4.2. Model Price v.s. Market Price: $T=360$ months, $P_{0}=96.83$.

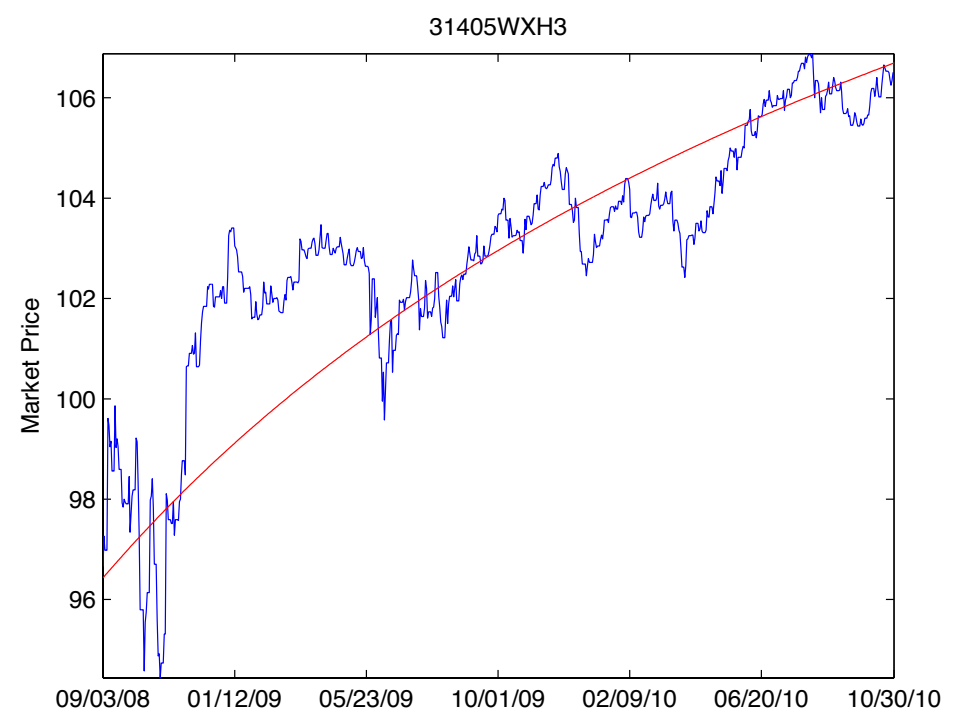

Fig. 4.3. Model Price v.s. Market Price: T=360 months, $P_{0}=96.41$.

4.1. Concluding remarks. In conclusion we have established that our reduced model possesses a strong solution. We have also numerically solved our model, and calibrated it to closely pick up a number of market trends observed immediately following the recessionary period of 2008. It would be an interesting question to consider $\rho \gg 1$. This case is important to address the subgroup of investors who want 


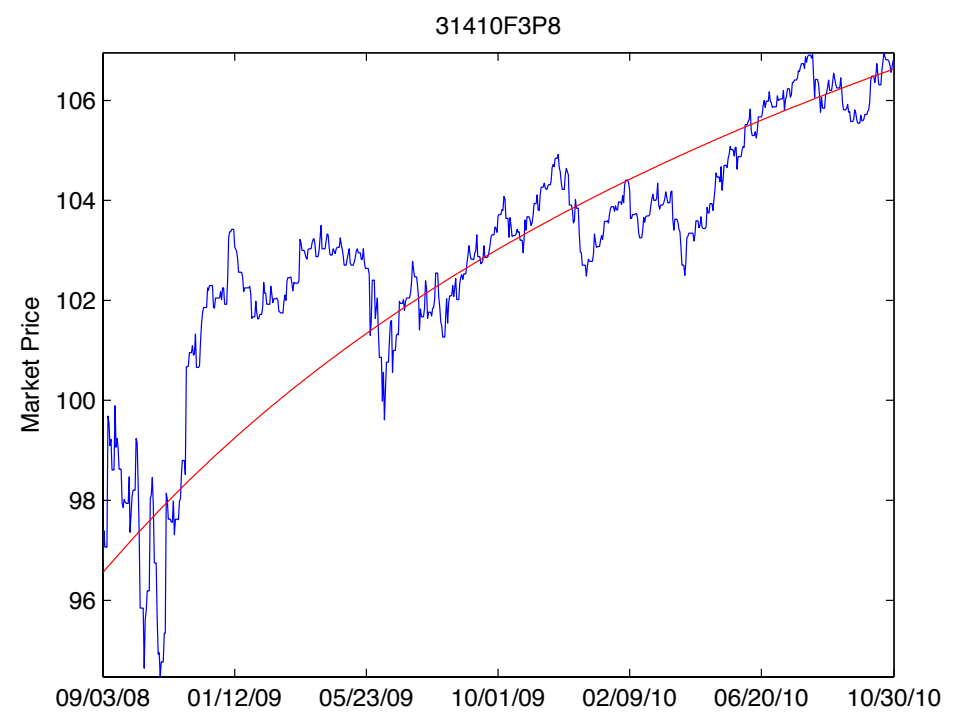

FIG. 4.4. Model Price v.s. Market Price: $T=180$ months, $P_{0}=96.53$.

less exposure to risk, as $\rho$ is essentially a measure of risk aversion. Alternatively, an interesting question would be to consider the behaviour of the reduced MBS model as $\rho \rightarrow 0$. This is the case of individuals who are extremely risk friendly. We would also like to point out that the ideal value of $\rho=1.25$, as found via calibration, makes the non linear term $\tilde{K}(V, t)$ behave essentially like $-V^{\frac{5}{3}}$, which actually contributes to the stability of the reduced PDE, and thus is a stabilising term. Furthermore, note that our proof of existence of mild and strong solutions requires the initial data to be in $H^{1}(U)$. It is still very much a challenge to prove existence of strong solutions if the initial data is just in $L^{2}(U)$. However, a benefit of our reduced model is to use it as a predictor, when one forsees economic scenarios consistent with the conditions, under which the model was derived in the first place, such as falling interest rates. To this end, we propose to experiment with different forms of the prepayment function in future work. It is our hope that the proposed simplified model is a step to gain some intuition behind the "breakdown" of the financial machinery, in the mortgage backed securities markets, over the recent recessionary period. We hope that this will open up further avenues for future research on one of the most pertinent economic issues of recent times.

Acknowledgements. This work is part of the first author's Masters project at Florida State University and an independent study undertaken under the supervision of Dr. Xiaoming Wang (the first authors PhD advisor), also at Florida State University. The first author would like to thank Dr. Yevgeny Goncharov for introducing him to the relevant PDE methods in the treatment of mortgage backed securities. 


\section{REFERENCES}

[1] F. Black and M. Scholes, The pricing of options and corporate liabilities, Journal of Political Economy, 81, 637-654, 1973.

[2] J.C. Butcher, The Numerical Analysis of Ordinary Differential Equations, Wiley-Interscience, 1987.

[3] J.W. Cooley and J.W. Tukey, An algorithm for the machine computation of the complex Fourier series, Math. Comput., 19, 297-301, 1965.

[4] M.G. Crandall, H. Ishii, and P.L. Lions, User's guide to viscosity solutions of second order partial differental equations, Bull. Amer. Math. Soc., 27, 1992.

[5] K. Dunn and J. McConnell, Valuation of GNMA mortgage backed securities, Journal of Finance, 36, 599-617, 1981.

[6] L.C. Evans, Partial Differential Equations, Graduate Studies in Mathematics, Providence, 19, 1998.

[7] F. Fabozzi, Handbook of Fixed Income Securities, John Wiley \& Sons, New Jersey, Hoboken, 2004.

[8] Y. Goncharov, Mathematical Theory of Mortgage Modelling, PhD dissertation, University of Chicago at Illinois, 2003.

[9] L.S. Hayre, Salomon Smith Barney Guide to Mortgage-Backed and Asset-Backed Securities, John Wiley \& Sons, New Jersey, Hoboken, 2001.

[10] P. Lattman, S. Patterson, and S. Ng, Wall street criticizes 'bad bank', The Wall Street Journal, 11 Feb, 2009, Web 20 Oct, 2009.

[11] R.D. Parshad, Asymptotic behaviour of the Darcy-Boussinesq system at large Darcy-Prandtl number, Disc. Cont. Dyn. Sys., Special Issue, 18, 26(4), 1441-1469, April 2010.

[12] R.D. Parshad, A reduced modelling approach to the pricing of mortgage backed securities, Elec. J. Diff. Equ., 136, 1-14, September, 2010.

[13] M. Papi, Viscosity Solutions and Optimization in Mathematical Finance, PhD Dissertation, Universita degli Studi di Roma Tor Vergata, 2003.

[14] M. Papi and M. Brianni, A PDE approach to pricing mortgage backed securities, Libera Universita Internazionale di Studi Sociale, Rome, preprint, 2004.

[15] N. Sharp, Advances in Mortgage Valuation: An Option Theoretic Approach, PhD Dissertation, University of Manchester, 2006.

[16] L. Trefethen, Spectral Methods in Matlab, SIAM, 2000.

[17] X. Wang, Asymptotic behavior of global attractors to the Boussinesq system for RayleighBenard convection at large prandtl number, Commun. Pure Appl. Math., 60, 1293-1318, 2007.

[18] G.R. Sell and Y. You, Dynamics of Evolutionary Equations, Appl. Math. Sci., Springer-Verlag, New York, 143, 2002.

[19] Bloomberg L.P.

[20] D. Nield and A. Bejan, Convection in Porous Media, $2^{\text {nd }}$ ed, Springer-Verlag, New York, 1999.

[21] M. Papi, Regularity results for a class of semilinear parabolic degenerate equations and applications, Commun. Math. Sci., 1(2), 229-244, 2003.

[22] X. Gabaix, A. Krishnamurty, and O.Vigneron, Limits of arbitrage: Theory and evidence from the mortgage-backed securities market, Journal of Finance, 2005.

[23] L.C. Rogers and D.Talay, Numerical Methods in Finance, Cambridge University Press, 1997.

[24] S.N. Neftci, Principles of Financial Engineering, Academic Press, Elsevier, 2008.

[25] A. O'Sullivan and S.M. Sheffrini, Economics: Principles in Action, Prentice Hall, 2003. 